\title{
DESCRIÇÃO DA QUALIDADE VOCAL DE PERSONAGENS IDOSOS DOS FILMES DE HOLLYWOOD
}

\section{Vocal quality description of senile characters from Hollywood movies}

\author{
Gisele Oliveira ${ }^{(1)}$, Mara Behlau ${ }^{(2)}$
}

\begin{abstract}
RESUMO
Objetivo: descrever a qualidade vocal de personagens idosos dos filmes de Hollywood. Métodos: foram colhidas 50 amostras de fala de personagens idosos, 11 do sexo feminino e 39 do masculino, de 38 filmes hollywoodianos dos anos de 1993 a 2001. Através da análise perceptivo-auditiva das amostras de fala, 20 fonoaudiólogos treinados classificaram cada personagem em idoso e não idoso, além de avaliarem as vozes quanto aos seguintes parâmetros citados pela literatura como mais alterados: rouquidão, crepitação, soprosidade, tensão, aspereza, astenia, nasalidade, tremor, modulação, pitch e estabilidade da frequência fundamental. Resultados: após a análise perceptivoauditiva, foi observado que a grande maioria dos atores (82\%) utilizou voz de idoso para representar seus papéis. O marcador mais evidente nas vozes foi alteração na qualidade vocal (92\%), demonstrada por crepitação $(80 \%)$, soprosidade $(54 \%)$, tensão $(38 \%)$, rouquidão $(30 \%)$ e astenia $(28 \%)$. 0 segundo marcador mais utilizado pelos atores nas suas representações foi a modulação vocal ampla e variada (44\%). Também foram observadas alterações no controle da voz (36\%) e instabilidade da frequência fundamental (38\%). Conclusão: a partir dos resultados obtidos pode-se concluir que os filmes de Hollywood caracterizam o idoso através de desvios evidentes na qualidade e modulação da voz, utilizando tipos de vozes alteradas e modulação vocal ampla e instável.
\end{abstract}

DESCRITORES: Voz; Qualidade da Voz; Envelhecimento

\section{INTRODUÇÃO}

A população idosa do mundo vem aumentando há algumas décadas e a tendência é de que essa porcentagem aumente ainda mais com o decorrer dos próximos anos.

Sem dúvida alguma pode-se dizer que houve grandes mudanças demográficas na população mundial, que já foi considerada jovem, porém esse não é o panorama atual. Na maioria dos países desenvolvidos a proporção da população com idade acima de 65 anos ultrapassa $10 \%$ do total. Nos EUA a população com idade acima de 65 anos é de $12,3 \%{ }^{1,2}$. Já no Brasil em 2000 , a população acima de 60 anos era de $8,6 \%{ }^{3}$.

(1) Fonoaudióloga; Vice-coordenadora do Curso de Especialização em Voz do Centro de Estudos da Voz, CEV, São Paulo, SP, Brasil; Doutoranda em Distúrbios da Comunicação na Universidade Federal de São Paulo.

(2) Fonoaudióloga; Diretora do Centro de Estudos da Voz, CEV, São Paulo, SP, Brasil; Doutora em Distúrbios da Comunicação pela Universidade Federal de São Paulo.

Conflito de interesses: inexistente
Alguns autores consideram apenas os aspectos cronológicos para classificar um indivíduo como idoso; outros por sua vez, consideram a terceira idade um processo basicamente biológico, indicando o declínio de várias funções vitais ${ }^{4,5}$. Apesar das divergências sobre o conceito, deve se ressaltar que a senescência é um processo universal, progressivo, irreversível e inevitável, e não pode ser vista como uma doença e ser estigmatizada, mas sim como modificações intrínsecas a todo ser vivo e inerentes à idade ${ }^{2,6,7}$.

Com a explosão da população idosa, profissionais da saúde têm procurado oferecer serviços especializados no tratamento de tais pacientes ${ }^{8,9}$. O conhecimento das mudanças normais tem se tornado mais importante do que nunca ${ }^{10,11}$.

O processo da senescência tem sido extensivamente pesquisado devido à importância do enveIhecimento de órgãos tão importantes e vitais, como coração, pulmão, cérebro e outros ${ }^{2,6,7}$. $O$ mesmo acontece na área de pesquisa da voz, com inúmeras investigações a respeito dos parâmetros anatômicos, fisiológicos, perceptivos e acústicos da voz 
senil ${ }^{12-27}$. No entanto, ainda há a necessidade de se obter dados normativos que representem valores que são esperados para indivíduos idosos.

A voz é o instrumento que leva consigo sentimentos e emoções, a qual se modifica de acordo com a idade, características físicas, situação, ambiente, história de vida, etc. O envelhecimento resulta num processo que afeta o perfeito funcionamento das estruturas responsáveis pela produção da fala ${ }^{2,12,28-32}$.

A sociedade moderna, principalmente no mundo ocidental, apregoa que o envelhecimento é perda de poder e possibilidades. O idoso é muitas vezes rotulado como frágil, confuso e ultrapassado, conceito este que faz a senescência ser temida e, muitas vezes adiada. Existe um apelo universal que leva o homem a buscar a juventude eterna, minimizando as rugas, tingindo os cabelos, fazendo exercícios físicos e lançando mão de técnicas mais ou menos agressivas que ofereçam uma imagem mais aceita pelo grupo social que o indivíduo está inserido.

Os meios de comunicação exercem influência muito grande na representação das várias classes e indivíduos da sociedade, além de possuir importante papel na divulgação de vários conceitos sociais. Embora exista uma crença popular generalizada de que a voz do idoso é estereotipada, esta regra não é comum na vida real, uma vez que pesquisas demonstram que ouvintes sem experiência são capazes de categorizar adequadamente falantes em dois grupos, jovens e idosos. Porém, existe uma percepção pública intuitiva que caracteriza a voz do idoso de uma forma errônea. A percepção pública é de que a voz na senescência é mais deteriorada do que é na realidade ${ }^{33}$. Portanto, um dos objetivos do presente trabalho é pesquisar a caracterização da voz do idoso realizada pelos filmes de Hollywood, uma vez que essas produções têm alcance mundial.

Von Leden ${ }^{34}$ afirma que apesar do estado de saúde mental e física de qualquer pessoa idosa, a comunicação verbal tende a se deteriorar com o avanço da idade. Mudanças vocais são virtualmente inevitáveis depois dos 60. Tais alterações nos órgãos da comunicação aumentam substancialmente em incidência e grau depois dos 70 anos de idade. $\mathrm{O}$ autor conclui que os efeitos do enveIhecimento no sistema expressivo da comunicação humana podem apresentar alterações no pitch, loudness e na qualidade vocal.

Há aspectos auditivos e acústicos que fazem parte das características da voz na terceira idade que são os marcadores do envelhecimento vocal, que sem dúvida alguma, auxiliam no processo da identificação da voz senil ${ }^{33,35-37}$. A voz do idoso é geralmente descrita como fraca ou astênica, soprosa, rouca, trêmula ${ }^{38,39}$. Alarcos, Behlau e Tosi 40 referem que o tremor é uma das características mais marcantes da voz na terceira idade e na deterioração vocal. Entre outras características estão a instabilidade da frequência fundamental e alteração de pitch e loudness.

O objetivo do presente trabalho é realizar uma descrição da qualidade vocal de personagens idosos dos filmes de Hollywood e verificar se por meio da voz o personagem é classificado como sendo idoso, por meio da análise perceptivo-auditiva de amostras de fala dos personagens.

\section{MÉTODOS}

Foram colhidas 50 amostras de fala de personagens idosos, 11 do sexo feminino e 39 do masculino, de 38 filmes hollywoodianos dos anos de 1993 a 2001. Foi estabelecido um período de 10 anos entre o filme mais antigo e o mais novo.

O critério de seleção de personagens foi a aparência física, uma vez que não é possível obter-se as idades dos mesmos. Foram inclusas amostras de fala de personagens representados tanto por atores idosos como por adultos.

As amostras foram retiradas a partir de fitas de vídeo cassete reproduzidas por um Video Cassete Recorder VR756/78 Philips e digitalizadas em um computador Dell, Dimension 4600. A média de duração das amostras foi de 15 segundos, com trechos de fala que apresentavam qualidade necessária para a avaliação auditiva.

As vozes foram submetidas à análise perceptivo-auditiva realizada por 20 fonoaudiólogos treinados, que foram instruídos a avaliar as amostras com base em um roteiro de avaliação desenvolvido para a pesquisa. A tarefa foi realizada em uma sala silenciosa e o material de voz apresentado por meio de caixas de som. Os resultados foram obtidos por consenso de dois terços do grupo. Amostras de fala foram repetidas tantas vezes quantas fossem necessárias para a obtenção do consenso do grupo. Num primeiro momento, os ouvintes classificaram auditivamente cada personagem em idoso e não idoso (Tabela 1). Em seguida foram analisados os seguintes parâmetros: rouquidão, crepitação, soprosidade, tensão, aspereza, astenia, nasalidade, tremor, modulação, pitch e estabilidade da frequência fundamental (Tabela 2).

Os dados foram submetidos a tratamento estatístico descritivo com cálculos de porcentagens. 


\section{RESULTADOS}

Os resultados da classificação perceptivo-auditiva da voz dos personagens em idoso ou não idoso podem ser observados na Tabela 1.

A análise perceptivo-auditiva das amostras de voz, de acordo com o sexo pode ser observada na Tabela 2.

\section{DISCUSSÃO}

Sem dúvida, pode-se dizer que não há outro processo natural que tenha maior impacto nas nossas vidas como o envelhecimento e com certeza, podese afirmar que a velhice traz consigo mudanças no corpo que geralmente modificam o som da voz.

Tabela 1 - Classificação perceptivo-auditiva da voz dos personagens idosos em idoso ou não idoso

\begin{tabular}{ccccc}
\hline \multirow{2}{*}{ Gênero } & \multicolumn{2}{c}{ Idoso } & N & Não Idoso \\
\cline { 2 - 6 } & $\mathbf{N}$ & $\%$ & 9 & $\%$ \\
\hline Masculino & 30 & 60 & 0 & 18 \\
Feminino & 11 & 22 & 9 & 0 \\
\hline TOTAL & 41 & 82 & 18 \\
\hline
\end{tabular}

Tabela 2 - Parâmetros perceptivo-auditiva da voz de personagens idosos dos filmes de Hollywood, de acordo com o sexo

\begin{tabular}{lcccccc}
\hline \multirow{2}{*}{ Parâmetros Vocais } & \multicolumn{2}{c}{ Masculino } & \multicolumn{2}{c}{ Feminino } & \multicolumn{2}{c}{ Total } \\
\cline { 2 - 7 } & $\mathbf{N}$ & $\%$ & $\mathbf{N}$ & $\%$ & $\mathbf{N}$ & $\%$ \\
\hline Qualidade Vocal & & & & & & \\
$\quad$ Rouquidão & 11 & 27 & 4 & 44 & 15 & 30 \\
Crepitação & 31 & 76 & 9 & 100 & 40 & 80 \\
Astenia & 8 & 20 & 6 & 67 & 14 & 28 \\
Soprosidade & 22 & 54 & 5 & 56 & 27 & 54 \\
Tensão & 15 & 37 & 4 & 44 & 19 & 38 \\
Pitch & & & & & & \\
$\quad$ agudo & 11 & 27 & 5 & 56 & 16 & 32 \\
$\quad$ grave & 13 & 32 & 1 & 11 & 14 & 28 \\
$\quad$ variável & 1 & 2 & 0 & 0 & 1 & 2 \\
Instabilidade & 24 & 59 & 8 & 89 & 32 & 64 \\
$\quad$ Modulação ampla & 16 & 29 & 6 & 67 & 22 & 44 \\
\hline
\end{tabular}

Há algumas décadas a senescência tem sido alvo de inúmeros estudos e pesquisas, uma vez que a população idosa no âmbito mundial teve um enorme crescimento ${ }^{1-3}$.

$\mathrm{Na}$ área da voz, o avanço das pesquisas tem colaborado para melhor entender este processo, todavia poucos estudos descrevem a voz do idoso. Portanto, a intenção deste estudo foi, não somente a de caracterizar auditivamente a voz na terceira idade, mas também de verificar a opinião, que um dos meios de comunicação mais abrangente como o cinema oferece ao público.

Algumas pesquisas demonstraram que é possível estimar com certa credibilidade a idade do falante através de sua voz ${ }^{33,35-37}$, porém a literatura também mostra que muitas vezes, ouvintes tendem a descrever a voz do idoso como sendo menos agradável e mais deteriorada do que é na realidade ${ }^{33}$.

Os atores hollywoodianos em sua grande maioria $(82 \%)$ representaram seus personagens idosos com voz característica à terceira idade. Os demais atores (18\%) apresentaram qualidade vocal fluida, sem alterações inerentes ao envelhecimento vocal (Tabela 1), o que provavelmente deve estar relacionado ao grau de experiência dos atores.

$\mathrm{O}$ envelhecimento é um conglomerado complexo de eventos biológicos que mudam a estru- 
tura e o funcionamento de várias partes do corpo. Entre tais mudanças estão velocidade, acurácia, tolerância, coordenação, capacidade respiratória, velocidade da condução nervosa, funcionamento cardíaco e renal. Tecidos musculares e nervosos, assim como ligamentos se atrofiam, substâncias químicas responsáveis pela transmissão nervosa sofrem modificações e as cartilagens se ossificam, inclusive as da laringe. Articulações ósseas desenvolvem irregularidades na sua movimentação. As pregas vocais ficam mais finas, deterioradas e perdem suas fibras elásticas e colágenas, o que as tornam mais rígidas, além de apresentarem suas bordas menos lisas ${ }^{2,12,14}$.

A literatura aponta para o fato de que o processo do envelhecimento ocorre de maneira e em períodos diferentes nos homens e nas mulheres. Há relatos de que as mudanças começam mais cedo no sexo masculino, e em muitos casos estas são mais drásticas do que as observadas no sexo feminino, ou seja, a deterioração vocal acontece em maior grau nos homens do que nas mulheres ${ }^{12,24,26,40}$.

A fonação normal é um minucioso mecanismo que exige um trajeto livre da onda mucosa das pregas vocais. Qualquer aspecto que interfira neste complexo percurso de vibração da onda mucosa pode resultar numa alteração vocal. Mudanças nas cartilagens, músculos, tecidos conectivos, glândulas e tecidos vasculares da laringe contribuem para mudanças no padrão de movimento das pregas vocais durante a produção da voz, e alteram a relação entre as variadas forças atuantes nesse refinado gerador de som. Tais alterações no funcionamento das estruturas afetam o som que é produzido durante a fonação, ou seja, sinais acústicos fazem com que os idosos carreguem tais mudanças impressas na sua voz. Portanto, pode-se presumir que o envelhecimento vocal possui marcadores específicos que garantem a identificação da voz do idoso. Tais mudanças histológicas, anatômicas e fisiológicas da laringe, certamente, resultarão em alterações tanto perceptivo-auditivas como acústicas da voz ${ }^{12-27}$.

Pesquisas anteriores mostram que o marcador mais evidente da voz do idoso é a alteração da qualidade vocal $4,9,19,20$ fato que corrobora os achados da presente pesquisa, demonstrado por crepitação $(80 \%)$, soprosidade $(54 \%)$, tensão $(38 \%)$, rouquidão $(30 \%)$ e astenia $(28 \%)$, como se observa na Tabela 2. Já o tremor, considerado o vilão na presbifonia, parece um artifício pouco (14\%) utilizado pelos atores de Hollywood para representar a voz senil ${ }^{38,40}$.

Devido às alterações musculares e respiratórias, é esperado que o idoso apresente alteração de loudness, o que resulta na qualidade vocal astênica ${ }^{17,21,34,38}$. Talvez pelo fato de uma produção vocal com intensidade reduzida, o que caracterizaria a voz astênica, exigir grande controle neuromuscular e respiratório, apenas $28 \%$ dos personagens apresentaram astenia.

Um outro grande marcador da voz na terceira idade é a alteração da frequência fundamental $2,16,17,19,20,21,24$. Com relação à frequência fundamental, as mulheres apresentam certa tendência em sua diminuição, já nos homens, ela tende a aumentar. A diminuição da $F_{0}$ nas mulheres pode ser explicada pelo edema e espessamento oriundos da alteração hormonal inerente à menopausa. Já o aumento da $F_{0}$ nos homens dá-se devido ao processo de calcificação e ossificação das cartilagens, e atrofia muscular. Isso não é o que ocorre com as vozes de idosos nos filmes, uma vez que $11 \%$ das vozes femininas apresentaram pitch grave, e $27 \%$ das vozes masculinas apresentaram pitch agudo. Tal achado pode demonstrar tanto que os atores não têm o conhecimento dessa alteração vocal ou que, simplesmente, não optam por utilizar esse ajuste para caracterizar a voz do idoso.

A literatura mostra que o indivíduo idoso apresenta dificuldades de entonação ${ }^{41}$, contudo os atores utilizaram modulação vocal ampla e variada (44\%), possivelmente porque durante a formação do ator, a modulação seja um parâmetro muito trabalhado e dito essencial na encenação. Embora o processo de envelhecimento vocal seja um evento inevitável, no ator ele pode, muitas vezes, ser menos evidente que em um indivíduo não-ator. Sabe-se que a atividade física continuada tem um efeito de minimização das mudanças causadas pela idade. Portanto, durante toda a sua carreira o ator submete seu sistema vocal a um uso intensivo, que pode minimizar e até mesmo postergar as alterações relacionadas ao avanço da idade ${ }^{12}$.

O controle da voz, ou seja, a instabilidade da $F_{0}$ (64\%) foi outro artifício bastante utilizado nos filmes e que aparece na literatura como característica da voz na senescência ${ }^{25-27,38,40}$.

Alteração de ressonância, mais evidenciada por um traço nasal na voz, também é apontada por estudos como sendo um marcador vocal na terceira idade ${ }^{2,27}$, contudo, não foi o que pôde-se observar nesta pesquisa, uma vez que, somente uma pequena porcentagem de vozes nasais $(8 \%)$ foram encontradas nas amostras de fala.

Embora a literatura ressalte que a sociedade possua uma ideia pré-concebida de que a voz senil é estereotipada; todos os achados obtidos através da avaliação perceptivo-auditiva no presente trabalho, apontam para o fato de que a voz do personagem idoso não se apresenta esteriotipada, além de que os filmes de Hollywood, na maioria 
das vezes, representam o idoso adequadamente através de sua voz.

\section{CONCLUSÕES}

A partir do estudo realizado com o objetivo de descrever a voz de 50 personagens idosos nos filmes de Hollywood, pode-se concluir que:
1) O personagem idoso apresenta voz como pertencente a um indivíduo idoso.

2) A voz do personagem idoso é de qualidade desviada, com maior ocorrência do tipo crepitante, soprosa, tensa, rouca e astênica, apresentando modulação vocal ampla e instabilidade de frequência fundamental.

\begin{abstract}
Purpose: to describe the vocal quality of Hollywood movies characters playing elderly people roles. Methods: a total of 50 aged character voice samples were used, 11 female and 39 male, from 38 Hollywood movies from the period between 1993 and 2001. Twenty speech therapists performed a perceptual auditory analysis. The listener's task required classifying each character either as elderly or as adult by their speech features, and also assessing their voices following the parameters that are most frequently addressed in the literature as being disordered at an old age. The parameters were hoarseness, vocal fry, breathiness, strain, roughness, vocal weakness, nasal resonance, tremor, vocal modulation, pitch and fundamental frequency stability. Results: after the perceptual auditory analysis we noted that a great number of actors (82\%) used senile voices indicating old age, even though not all of them were elderly. The most salient voice set altered was vocal quality (92\%), demonstrated by vocal fry $(80 \%)$, breathiness $(54 \%)$, strain $(38 \%)$, hoarseness $(30 \%)$, and weak voice $(28 \%)$. The second vocal set most used by the actors for their roles was extensive vocal modulation (44\%). It was also observed that vocal instability $(64 \%)$ was used by the actors. Conclusion: it can be concluded from the results that Hollywood movies characterize the elderly characters through evident deviations in vocal quality and modulation, using disordered types of voices and vocal unstable and extensive modulation.
\end{abstract}

KEYWORDS: Voice; Vocal Quality; Aging

\section{REFERÊNCIAS}

1. U.S. Census Bureau, Current Population Survey, Annual Social and Economic Supplement, 2008. [citado 2009 Jul 07]. Disponível em: http:// www.census.gov/population/www/socdemo/age/ older_2008.html

2. Linville SE. Vocal aging. Singular: San Diego; 2000.

3. IBGE. Instituto Brasileiro de Geografia e Estatística. [citado 2009 Jul 07]. Disponível em: http://www.ibge.gov.br/home/estatistica/populacao/ perfilidoso/tabela1_2.shtm

4. Bengtson VL, Silverstein M, Putney N. Theories about age and aging. In: Bengtson VL, Gans D, Putney N, Silverstein M. Handbook of theories of aging. 2.ed.New York: Springer. p.3-20.

5. Schneider $\mathrm{RH}$, Irigaray $\mathrm{TQ}$. O envelhecimento na atualidade: aspectos cronológicos, biológicos, psicológicos e sociais. Estud. Psicol. 2008; 25(4):585-93.
6. Charchat-Fichman H, Caramelli P, Sameshima K, Nitrini R. Declínio da capacidade cognitiva durante o envelhecimento. Rev. Bras. Psiquiatr. 2005; 27(1):79-82.

7. Teixeira LA. Declínio de desempenho motor no envelhecimento é específico à tarefa. Rev Bras Med Esporte. 2006; 12(6):351-5.

8. Motta LB, Aguiar AC. Novas competências profissionais em saúde e o envelhecimento populacional brasileiro: integralidade, interdisciplinaridade e intersetorialidade. Ciênc. Saúde Coletiva. 2007; 12(2):363-72.

9. Silva LRF. Da velhice à terceira idade: o percurso histórico das identidades atreladas ao processo de envelhecimento. Hist. Cienc. Saúde-Manguinhos. 2008; 15(1):155-68.

10. Prado SD, Sayd JD. A pesquisa sobre envelhecimento humano no Brasil: grupos e linhas de pesquisa. Ciênc. Saúde Coletiva. 2004; 9(1):57-68. 
11. Cupertino APFB, Rosa FHM, Ribeiro PCC. Definição de envelhecimento saudável na perspectiva de indivíduos idosos. Psicol. Reflex. Crit. 2007; 20(1):81-6.

12. Sataloff RT, Linville E. The effects of age on the voice. In: Sataloff RT. Professional voice: the science and art of clinical care. Vol 2. 3. ed. Plural: San Diego; 2005. p.497-511.

13. Thomas LB, Harrison AL, Stemple JC. Aging thyroarytenoid and limb skeletal muscle: lessons in contrast. J Voice. 2008; 22(4):430-50.

14. Pontes $P$, Yamasaki R, Behlau M. Morphological and functional aspects of the senile larynx. Folia Phoniatr Logop. 2006; 58(3):151-8. http://dx.doi. org/10.1159/000091729

15. Pontes P, Brasolotto A, Behlau M. Glottic characteristics and voice complaint in the elderly. $J$ Voice. 2005; 19(1):84-94.

16. Awan SN. The aging female voice: acoustic and respiratory data. Clin Linguist Phon. 2006; 20(2-3):171-80. http://dx.doi. org/10.1080/02699200400026918

17. Cerceau JSB, Alves CFT, Gama ACC. Análise acústica da voz de mulheres idosas. Rev. CEFAC. 2009; 11(1):142-9. http://dx.doi.org/10.1590/ S1516-18462009005000017

18. Huber JE, Spruill J III. Age-related changes to speech breathing with increased vocal loudness. J Speech Lang Hear Res. 2008; 51(3):651-68.

19. Nishio M, Niimi S. Changes in speaking fundamental frequency characteristics with aging. Folia Phoniatr Logop. 2008; 60(3):120-7. http:// dx.doi.org/10.1159/000118510

20. Vongpaisal T, Pichora-Fuller MK. Effect of age on FO difference limen and concurrent vowel identification. J Speech Lang Hear Res. 2007; 50(5):1139-56. http://dx.doi.org/10.1044/1092-4388 (2007/079)

21. Decoster W, Debruyne F. Longitudinal voice changes: facts and interpretation. J Voice. 2000; 14(2):184-93.

22. Winkler R, Sendlmeier W. EGG open quotient in aging voices-changes with increasing chronological age and its perception. Logop Phoniatr Vocol. 2006; 31(2):51-6. http://dx.doi. org/10.1080/14015430500445534

23. Makiyama K, Yoshihashi H, Park R, Shimazaki $\mathrm{N}$, Nakai M. Assessment of phonatory function by the airway interruption method: age-related changes. Otolaryngol Head Neck Surg. 2006; 134(3):407-12. http://dx.doi.org/10.1016/j.otohns.2005.11.009
24. Iseli M, Shue YL, Alwan A. Age, sex, and vowel dependencies of acoustic measures related to the voice source. J Acoust Soc Am. 2007; 121(4):2283-95.

25. Gorham-Rowan MM, Laures-Gore J. Acousticperceptual correlates of voice quality in elderly men and women. J Commun Disord. 2006; 39(3):171-84.

26. Soyama CK, Espassatempo CL, Gregio FN, Camargo Z. Qualidade vocal na terceira idade: parâmetros acústicos de longo termo de vozes masculinas e femininas. Rev. CEFAC. 2005; 7(2):267-79.

27. Linville SE, Fisher HB. Acoustic characteristics of perceived versus actual vocal age in controlled phonation by adult females. J Acoust Soc Am. 1985; 78(1Pt1):40-8.

28. Kendall K. Presbyphonia: a review. Curr Opin Otolaryngol Head Neck Surg. 2007; 15(3):137-40.

29. Baken RJ. The aged voice: a new hypothesis. J Voice. 2005; 19(3):317-25.

30. Verdonck-de Leeuw IM, Mahieu H. Vocal aging and the impact on daily life: a longitudinal study. J Voice. 2004; 18(2):193-202.

31. Menezes LN, Vicente LCC. Envelhecimento vocal em idosos instucionalizados. Rev. CEFAC. 2007; 9(1):90-8. http://dx.doi.org/10.1590/ S1516-18462007000100012

32. Gampel D, Karsch UM, Ferreira LP. Envelhecimento, voz e atividade física de professores e não professores. Rev. Soc. Bras. Fonoaudiol. 2008; 13(3):218-25.

33. Huntley R, Hollien H, Shipp T. Influences of listener characteristics on perceived age estimations. J Voice. 1987; 1(1):49-52.

34. Von Leden H. Speech and hearing problems in the geriatric patient. J Am Geriatr Soc. 1977; 25(9):422-6.

35. Harnsberger JD, Shrivastav R, Brown WS $\mathrm{Jr}$, Rothman $\mathrm{H}$, Hollien $\mathrm{H}$. Speaking rate and fundamental frequency as speech cues to perceived age. J Voice. 2008; 22(1):58-69.

36. Neiman GS, Applegate JA. Accuracy of listener judgments of perceived age relative to chronological age in adults. Folia Phonitr Logop. 1990; 42(6):32730. http://dx.doi.org/10.1159/000266090

37. Rossi-Katz J, Arehart KH. Message and talker identification in older adults: effects of task, distinctiveness of the talkers' voices, and meaningfulness of the competing message. J Speech Lang Hear Res. 2009; 52(2):435-53.

38. Casper JK, Colton RH. Current understanding and treatment of phonatory disorders in geriatric populations. Curr Opin Otolaryngol Head Neck Surg. 2000; 8(3):158-64. 
39. Polido AM, Martins MASUR, Hanayama EM. Percepção do envelhecimento vocal na terceira idade. Rev. CEFAC. 2005; 7(2):241-51.

40. Alarcos AL, Behlau MS, Tosi O. Computer acoustical analysis of senile voices. [letter] Folia Phoniatr.1983; 35:102.
41. Fecteau S, Armony JL, Joanette $\mathrm{Y}$, Belin $P$. Judgment of emotional nonlinguistic vocalizations: age-related differences. Appl Neuropsychol. 2005; 12(1):40-8. http://dx.doi.org/ 10.1207/s15324826an1201_7

RECEBIDO EM: 27/02/2009

ACEITO EM: 22/12/2009

Endereço para correspondência:

Gisele Oliveira

Rua Machado Bittencourt, $36110^{\circ}$ andar conj. 1005

São Paulo - SP

CEP: 04044-001

E-mail: giseleoliveiracev@uol.com.br 